\title{
Exterior complex scaling method in time-dependent density-functional theory: Multiphoton ionization and high-order-harmonic generation of Ar atoms
}

\author{
Dmitry A. Telnov, ${ }^{1, *}$ Ksenia E. Sosnova, ${ }^{1}$ Efim Rozenbaum, ${ }^{1}$ and Shih-I Chu ${ }^{2, \dagger}$ \\ ${ }^{1}$ Department of Physics, St. Petersburg State University, St. Petersburg 198504, Russia \\ ${ }^{2}$ Department of Chemistry, University of Kansas, Lawrence, Kansas 66045, USA
}

(Received 3 March 2013; published 14 May 2013)

\begin{abstract}
The exterior complex scaling (ECS) method is applied in the framework of time-dependent density-functional theory (TDDFT) to study multiphoton ionization (MPI) and high-order-harmonic generation (HHG) of multielectron atoms in intense laser fields. ECS allows one to impose correct (outgoing-wave) boundary conditions on the wave functions at large distances. In our implementation, ECS is combined with the time-dependent generalized pseudospectral method for accurate and efficient solution of the time-dependent Kohn-Sham equations. We make use of LB94 exchange-correlation potential which proved accurate in calculations of unperturbed electronic structure of Ar. Calculations of MPI and HHG are performed for the laser pulses with the wavelength of $800 \mathrm{~nm}$ and several peak intensities. The HHG spectrum exhibits an intensity-independent minimum corresponding to the photon energy of about $51 \mathrm{eV}$ which is closely related to the Cooper minimum observed in the photoionization cross section of Ar. We found that MPI probabilities and HHG spectra calculated with the frozen-core potential (that is, not including dynamic response of the electron density) differ significantly from those obtained by TDDFT.
\end{abstract}

DOI: 10.1103/PhysRevA.87.053406

PACS number(s): 32.80.Rm, 42.65.Ky, 31.15.ee

\section{INTRODUCTION}

Strong-field multiphoton processes such as multiphoton ionization (MPI) and high-order-harmonic generation (HHG) continued attracting much interest in recent years both experimentally and theoretically due to advances in laser technology that marked the birth of attosecond physics [1]. In particular, tunable long-wavelength lasers have become available; they provide sufficiently high intensities without saturation of ionization thus probing both valence and core electrons. MPI and HHG processes have a capability of imaging of atomic and molecular structures with high resolution in spatial and temporal domains [2-4]. Electronic structure of atoms and molecules can be encoded in the HHG signal; the latter may contain features due to collective multielectron effects involving inner-shell electrons $[3,5]$. The multielectron structural information can be retrieved by means of the HHG interferometry which is established as an effective approach to resolving multielectron dynamics [3].

Although most of the atomic and molecular targets are multielectron systems, traditionally many theoretical studies of MPI and HHG are based on various implementations of the single active electron (SAE) model and strong-field approximation (SFA). However, while SFA theories result in rather simple analytical expressions, they fail to give a quantitative description of the processes. The discrepancy with more accurate approaches or direct solutions of the time-dependent Schrödinger equation can be as large as several orders of magnitude. On the contrary, SAE models can be quite accurate (see, for example, Refs. [6,7] where the SAE calculations were successfully compared with the experiment on above-threshold ionization of argon atoms), but they depend very much on the state-of-the-art effective potentials specific

\footnotetext{
*telnov@pcqnt1.phys.spbu.ru

†sichu@ku.edu
}

for each atomic or molecular system. SFA and SAE theories usually deal with the highest-occupied orbitals only and neglect the multielectron dynamics of the target atoms and molecules. However, multielectron effects due to the electron exchange and correlation may be significant even when the inner electrons are strongly bound and are not excited by the driving laser field. Our theoretical method is based on the extension of time-dependent density-functional theory (TDDFT) with proper long-range potentials (for a review, see [8]). The method takes into account the dynamic response of all the electronic shells to the external fields and has been applied successfully to the nonperturbative study of MPI and HHG of atoms and diatomic molecules [9-11] in intense laser fields.

Atomic systems subject to laser fields can be ionized. At large distances from the core, only outgoing-wave components (describing ionization) should be present in the wave function. Thus the correct boundary conditions are the outgoing-wave boundary conditions, if ionization takes place. Boundary conditions can be imposed by an absorber placed at some distance from the core. The absorber prevents the electron density from moving back to the core thus imposing the outgoing-wave boundary conditions. However, the results (ionization probability and HHG spectra) depend on the properties of the absorber.

Another way to impose correct boundary conditions is to apply a complex-scaling transformation $[12,13]$. The complexscaled wave function is supposed to vanish at infinity in the coordinate space, which means the unscaled wave function must satisfy the outgoing-wave boundary conditions. Uniform complex scaling, however, is not suitable for time-dependent problems since the external field changes sign twice per optical cycle. Thus the complex-scaled propagator may diverge at large distances. Exterior complex scaling can solve the problem. Dipole interaction with external field can be applied in the interior (not complex-scaled) domain only which should 
be chosen large enough to include all physically important regions.

Exterior complex scaling (ECS) $[14,15]$ may have advantages when applied to more complex systems with potentials which behave nonanalytically (or defined only numerically) in the interior region of the coordinates. ECS procedure assumes that the coordinate space is partitioned in two domains, and the complex scaling is applied in the exterior domain only, while in the interior domain the wave function remains unchanged. The derivative of the ECS mapping function may appear continuous or discontinuous on the boundary between the two regions; these two cases are termed smooth ECS and sharp ECS, respectively. Previous implementations of ECS in theoretical atomic and molecular physics were mostly related to time-independent photoionization [16] and scattering [17] problems (see also the review paper [18]). For the timedependent Schrödinger equation, complex finite elements schemes have been used to study propagation of wave packets in one dimension [19]. Time-dependent approaches have also been used for construction of time-independent scattering wave functions [20]. In our previous studies, we applied sharp ECS to time-independent Floquet eigenvalue problems which arise in one-electron model potential calculations [21] and Floquet formulation of TDDFT [22] (see also the review article [23]).

The organization of the paper is as follows. In Sec. II, we describe our implementation of smooth ECS in generalized pseudospectral method. In Sec. III, we give the necessary details of the Kohn-Sham DFT scheme for the calculation of the electronic structure of Ar atoms. Section IV contains a description of the time-dependent computational scheme which combines the TDDFT and ECS approaches. In Sec. V, we present the results of the calculations of multiphoton ionization and high-order-harmonic generation of $\mathrm{Ar}$ atoms. Section VI contains a summary of our results.

\section{SMOOTH ECS IMPLEMENTATION IN GENERALIZED PSEUDOSPECTRAL METHOD}

To solve the time-dependent and time-independent Schrödinger or Kohn-Sham equations, we apply the generalized pseudospectral (GPS) discretization of wave functions and operators in spherical polar coordinates. Detailed description of the GPS discretization is given elsewhere [24,25] using prolate spheroidal coordinates in the case of two-center systems. Here we list basic equations pertinent to the present case of spherical coordinates. The radial coordinate $r$ is discretized using the Gauss-Lobatto scheme where the collocation points are the roots of the polynomial $\left(1-x^{2}\right) P_{N_{x}}^{\prime}(x), P_{N_{x}}^{\prime}(x)$ being the derivative of the Legendre polynomial. There are $N_{x}+2$ collocation points within the interval $[-1,1]$, including the points -1 and 1 . The latter two points correspond to the boundaries of the radial coordinate range when an appropriate mapping between $x$ and $r$ is established. If zero boundary conditions are imposed on the wave function at these points, only $N_{x}$ internal collocation points are used to represent the discretized wave function.

The mapping function $r(x)$ and its two derivatives must be continuous to make sure the wave function and its derivatives are continuous, too. We adopt the following mapping transformation:

$$
r=r(x), \quad r(x)=R(x) \exp [i \alpha(x)],
$$

where $R(x)$ is a real monotonous function which maps the interval $[-1,1]$ to the radial coordinate range $\left[0, R_{b}\right]$ used to solve the equations:

$$
R(x)=R_{m} \frac{(1+x)^{2}+2 \delta(1+x)}{1-x+4 R_{m}(1+\delta) / R_{b}} .
$$

Here $R_{m}, R_{b}$, and $\delta$ are parameters of the transformation. The end point $R_{b}$ must be chosen large enough to ensure the space domain used to solve the equation contains all important physics. In our calculations for the Ar atom, we use the following values of the parameters:

$$
R_{m}=30 \text { a.u., } \quad R_{b}=200 \text { a.u. }, \quad \delta=0.02 .
$$

A quadratic term in the numerator of Eq. (2) and a small value of the parameter $\delta$ make the distribution of the radial grid points denser in the vicinity of the nucleus; this is important for improving the accuracy of the calculations since the argon nucleus charge is quite large, and the inner shell wave functions vary rapidly on small distances around the nucleus.

The complex rotation of the radial coordinate is controlled by the phase $\alpha(x)$ in Eq. (1). We use the following piecewise polynomial dependence of $\alpha$ on $x$ :

$$
\begin{aligned}
\alpha(x)= & 0, \quad-1 \leqslant x \leqslant x_{0} ; \\
\alpha(x)= & \frac{10 \alpha_{0}}{\left(x_{1}-x_{0}\right)^{5}}\left[\left(x-x_{0}\right)^{3}\left(x_{1}-x\right)^{2}\right. \\
& \left.+\frac{1}{2}\left(x-x_{0}\right)^{4}\left(x_{1}-x\right)+\frac{1}{10}\left(x-x_{0}\right)^{5}\right], \\
x_{0} \leqslant & x \leqslant x_{1} ; \quad \alpha(x)=\alpha_{0}, \quad x_{1} \leqslant x \leqslant 1 .
\end{aligned}
$$

As one can see, $\alpha(x)$ along with its first and second derivatives are continuous functions at the boundary points $x_{0}$ and $x_{1}$. In the interior domain $x<x_{0}, \alpha(x)=0$; within the range $\left[x_{0}, x_{1}\right], \alpha(x)$ gradually increases to reach the value $\alpha_{0}$ at $x=x_{1}$; in the asymptotic region $x>x_{1}$, the complex rotation angle is equal to $\alpha_{0}$. Its first derivative has a simple form within the middle interval $\left[x_{0}, x_{1}\right]$ :

$$
\frac{d \alpha}{d x}=\frac{30 \alpha_{0}}{\left(x_{1}-x_{0}\right)^{5}}\left(x-x_{0}\right)^{2}\left(x-x_{1}\right)^{2}, \quad x_{0} \leqslant x \leqslant x_{1} .
$$

In the coordinate space, the radius of the interior domain, $R_{0} \equiv$ $R\left(x_{0}\right)$, should be sufficiently large to accommodate oscillations in the laser field of a free electron emerging in the vicinity of the nucleus. For weaker laser fields (peak intensity up to $2 \times 10^{14} \mathrm{~W} / \mathrm{cm}^{2}$ ), we set $R_{0}=25$ a.u., $R_{1} \equiv R\left(x_{1}\right)=60$ a.u., and $\alpha_{0}=0.8$; the corresponding ECS contour in the complex plane of the radial coordinate $r$ is shown in Fig. 1. For stronger fields, larger values of $R_{0}=30$ a.u. and $R_{1}=65$ a.u. have been used. The number of the radial grid points $N_{x}$ for weaker and stronger fields was 256 and 320, respectively.

For the polar angle $\vartheta$, we discretize the variable $\cos \vartheta$ using the Gauss scheme where the collocation points are the roots of the Legendre polynomial $P_{N_{y}}(y)$. There are $N_{y}$ collocation points which lie entirely within the interval $[-1,1]$. Since $\cos \vartheta$ spans the same range, a simple identity mapping can be 


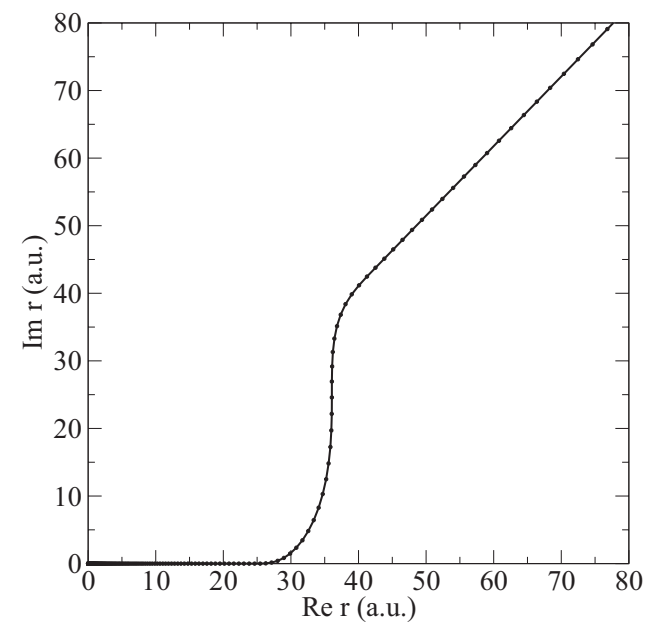

FIG. 1. ECS contour in the complex plane of the radial coordinate $r$ for $R\left(x_{0}\right)=25$ a.u., $R\left(x_{1}\right)=60$ a.u., and $\alpha_{0}=0.8$. Filled circles indicate positions of the radial grid points.

used between $\cos \vartheta$ and $y$ :

$$
\cos \vartheta=y .
$$

For linearly polarized laser fields, the projection $m$ of the angular momentum on the polarization direction of the field is conserved (we assume that the unperturbed Hamiltonian is spherically symmetric). For $m$ fixed, the dependence of the wave function $\Psi(\boldsymbol{r}, t)$ on the azimuthal angle $\varphi$ is given by the analytical factor

$$
\Psi(\boldsymbol{r}, t)=\psi(r, \vartheta, t) \exp (i m \varphi)
$$

and does not require numerical representation. The remaining factor $\psi(r, \vartheta, t)$ is discretized on the two-dimensional grid $\left[r_{i}=\right.$ $r\left(x_{i}\right)$ and $\left.\vartheta_{j}=\vartheta\left(y_{j}\right)\right]$. It is convenient to introduce an array $\phi_{i j}$ containing scaled values of $\psi\left(r_{i}, \vartheta_{j}, t\right)$ :

$$
\begin{aligned}
\psi\left(r_{i}, \vartheta_{j}, t\right)= & \phi_{i j} \sqrt{\frac{\left(N_{x}+1\right)\left(N_{x}+2\right)}{8 \pi r_{i}^{\prime}}} \frac{\sin \vartheta_{j}}{r_{i}} \\
& \times P_{N_{x}+1}\left(x_{i}\right) P_{N_{y}}^{\prime}\left(y_{j}\right)
\end{aligned}
$$

( $r_{i}^{\prime}$ denotes the values of the derivative $d r / d x$ at the collocation points $x_{i}$ ). Then the normalization integral of the wave function is calculated as follows:

$$
\int d^{3} r|\Psi(\boldsymbol{r}, t)|^{2}=\sum_{i=1}^{N_{x}} \sum_{j=1}^{N_{y}}\left|\phi_{i j}\right|^{2} .
$$

The kinetic energy operator is represented by a matrix acting on the vector $\phi_{i j}$. Different forms of the kinetic energy matrix are obtained for $m=0$ and $m=1$ (higher angular momentum projections are irrelevant for Ar):

$$
\begin{aligned}
T_{i j, i^{\prime} j^{\prime}}= & \frac{1}{2}\left[\frac{\delta_{j j^{\prime}}}{\sqrt{r_{i}^{\prime} r_{i^{\prime}}^{\prime}}} \sum_{k=0}^{N_{x}+1} \frac{d_{k i}^{x} d_{k i^{\prime}}^{x}}{r_{k}^{\prime}}\right. \\
& \left.+\frac{\delta_{i i^{\prime}} \sin \vartheta_{j} \sin \vartheta_{j^{\prime}}}{r_{i}^{2}} \sum_{k=1}^{N_{y}} d_{k j}^{y} d_{k j^{\prime}}^{y}\right] \quad(m=0),
\end{aligned}
$$

$$
\begin{aligned}
T_{i j, i^{\prime} j^{\prime}}= & \frac{\delta_{i i^{\prime}} \delta_{j j^{\prime}}}{r_{i}^{2}}+\frac{1}{2}\left[\frac{\delta_{j j^{\prime}}}{\sqrt{r_{i}^{\prime} r_{i^{\prime}}^{\prime}}} \sum_{k=0}^{N_{x}+1} \frac{d_{k i}^{x} d_{k i^{\prime}}^{x}}{r_{k}^{\prime}}\right. \\
& \left.+\frac{\delta_{i i^{\prime}}}{r_{i}^{2}} \sum_{k=1}^{N_{y}} \sin ^{2} \vartheta_{k} d_{k j}^{y} d_{k j^{\prime}}^{y}\right] \quad(m=1),
\end{aligned}
$$

where the first derivative matrices $d_{i i^{\prime}}^{x}$ and $d_{j j^{\prime}}^{y}$ are defined as follows:

$$
\begin{gathered}
d_{i i^{\prime}}^{x}=\frac{1}{x_{i}-x_{i^{\prime}}}\left(i \neq i^{\prime}\right), d_{i i}^{x}=0\left(1 \leqslant i \leqslant N_{x}\right) \\
d_{j j^{\prime}}^{y}=\frac{1}{y_{j}-y_{j^{\prime}}}\left(j \neq j^{\prime}\right), d_{j j}^{y}=\frac{y_{j}}{1-y_{j}^{2}} .
\end{gathered}
$$

All potential terms are represented by their values on the coordinate grid and appear as diagonal matrices in the GPS method; no calculation of potential energy matrix elements is required.

\section{ELECTRONIC STRUCTURE CALCULATIONS OF ARGON ATOMS}

As a first step of our calculations, we solve the set of timeindependent Kohn-Sham equations for the unperturbed spin orbitals $\psi_{n \sigma}(\boldsymbol{r})$ and spin-orbital energies $\epsilon_{n \sigma}$ :

$$
\begin{aligned}
{\left[-\frac{1}{2} \nabla^{2}+V_{\sigma}^{0}(\boldsymbol{r})\right] \psi_{n \sigma}(\boldsymbol{r}) } & =\epsilon_{n \sigma} \psi_{n \sigma}(\boldsymbol{r}), \\
n & =1,2, \ldots, N_{\sigma} .
\end{aligned}
$$

Here $N_{\sigma}\left(=N_{\uparrow}\right.$ or $\left.N_{\downarrow}\right)$ is the total number of electrons for a given spin $\sigma$; the total number of electrons in the system is $N=N_{\uparrow}+N_{\downarrow}$. The electron spin densities $\rho_{\sigma}(\boldsymbol{r})$ and the total density $\rho(\boldsymbol{r})$ are related to the spin orbitals as follows:

$$
\begin{aligned}
& \rho_{\sigma}(\boldsymbol{r})=\sum_{n=1}^{N_{\sigma}}\left|\psi_{n \sigma}(\boldsymbol{r})\right|^{2}, \\
& \rho(\boldsymbol{r})=\rho_{\uparrow}(\boldsymbol{r})+\rho_{\downarrow}(\boldsymbol{r}),
\end{aligned}
$$

and the effective single-particle potential $V_{\sigma}^{0}(\boldsymbol{r})$ can be represented as a sum of three different contributions:

$$
V_{\sigma}^{0}(\boldsymbol{r})=v_{\mathrm{n}}(\boldsymbol{r})+v_{\mathrm{H}}(\boldsymbol{r})+v_{\mathrm{xc}, \sigma}(\boldsymbol{r}) .
$$

Here $v_{\mathrm{n}}(\boldsymbol{r})$ is a Coulomb interaction of the electron with the nucleus,

$$
v_{\mathrm{n}}(\boldsymbol{r})=-\frac{Z}{r},
$$

with $Z=18$ being the nuclear charge; $v_{\mathrm{H}}(\boldsymbol{r})$ is the Hartree potential due to electron-electron repulsion,

$$
v_{\mathrm{H}}(\boldsymbol{r})=\int \frac{\rho\left(\boldsymbol{r}^{\prime}\right) d^{3} r^{\prime}}{\left|\boldsymbol{r}-\boldsymbol{r}^{\prime}\right|} .
$$

The remaining term $v_{\mathrm{xc}, \sigma}(\boldsymbol{r})$ is the exchange-correlation potential. Its exact form is unknown but high-quality approximations are becoming available. In this work, we make use of an approximate exchange-correlation potential LB94 by van 
TABLE I. Absolute values of spin-orbital energies of Ar. (A) Present DFT calculations (eV). (B) Experimental binding energies (eV) [27].

\begin{tabular}{lrr}
\hline \hline Spin orbital & A & \multicolumn{1}{c}{ B } \\
\hline $1 s$ & 3158.8 & 3206.3 \\
$2 s$ & 311.7 & 326.3 \\
$2 p$ & 247.0 & 248.5 \\
$3 s$ & 29.3 & 29.3 \\
$3 p$ & 15.7 & 15.8 \\
\hline \hline
\end{tabular}

Leeuwen and Baerends [26]:

$$
\begin{aligned}
v_{\mathrm{xc}, \sigma}^{\mathrm{LB} 94}(\boldsymbol{r})= & v_{\mathrm{x}, \sigma}^{\mathrm{LSDA}}(\boldsymbol{r})+v_{\mathrm{c}, \sigma}^{\mathrm{LSDA}}(\boldsymbol{r}) \\
& -\frac{\beta x_{\sigma}^{2}(\boldsymbol{r}) \rho_{\sigma}^{1 / 3}(\boldsymbol{r})}{1+3 \beta x_{\sigma}(\boldsymbol{r}) \ln \left\{x_{\sigma}(\boldsymbol{r})+\left[x_{\sigma}^{2}(\boldsymbol{r})+1\right]^{1 / 2}\right\}} .
\end{aligned}
$$

Here $v_{\mathrm{x}, \sigma}^{\mathrm{LSA}}(\boldsymbol{r})$ and $v_{\mathrm{c}, \sigma}^{\mathrm{LSDA}}(\boldsymbol{r})$ are the exchange and correlation potentials in the local spin-density approximation (LSDA), respectively, and $x_{\sigma}(\boldsymbol{r})=\left|\nabla \rho_{\sigma}(\boldsymbol{r})\right| / \rho_{\sigma}^{4 / 3}(\boldsymbol{r})$. The last term in Eq. (20) depends on the parameter $\beta$; its value $(\beta=0.05)$ was adjusted to fit the exact exchange-correlation potential for the beryllium atom [26]. As our results show, this potential is also very accurate in calculations of the electronic structure of argon atoms. Since Ar is a closed-shell atom, the electron densities for spin up and spin down are the same, and the set of equations (14) can be solved for one spin projection only (either spin up or spin down). The equations are solved self-consistently, starting from some reasonable approximation for the potential $V_{\sigma}^{0}(\boldsymbol{r})$, until convergence is achieved.

We list the orbital energies in Table I and compare them with the experimental binding energies. As one can see, the accuracy is very good for $2 p, 3 s$, and $3 p$ energy levels. In our calculations, we do not take into account the spin-orbit interaction. Experimental observations do resolve spin-orbit splitting of $p_{1 / 2}$ and $p_{3 / 2}$ energy levels; it constitutes about $1 \%$, which is the same order as the accuracy limit of our calculations. In Table I, we show the experimental binding energies for the $p_{3 / 2}$ sublevels only. For the inner shells ( $1 s$ and $2 s$ ), deviation of our results from the experiment is somewhat larger but still does not exceed $5 \%$.

\section{TDDFT-ECS CALCULATIONS FOR ARGON ATOMS}

For the atoms subject to external time-dependent fields, we apply TDDFT and solve the set of time-dependent Kohn-Sham equations:

$$
\begin{aligned}
i \frac{\partial}{\partial t} \psi_{n \sigma}(\boldsymbol{r}, t) & =H(t) \psi_{n \sigma}(\boldsymbol{r}, t), \quad n=1,2, \ldots, N_{\sigma} ; \\
H(t) & =-\frac{1}{2} \nabla^{2}+V_{\sigma}^{0}(\boldsymbol{r}, t)+v_{\mathrm{ext}}(\boldsymbol{r}, t) .
\end{aligned}
$$

The potential $v_{\text {ext }}(\boldsymbol{r}, t)$ in Eq. (22) describes the interaction with the laser field. Using the dipole approximation and the length gauge, it can be expressed as follows:

$$
v_{\mathrm{ext}}(\boldsymbol{r}, t)=\boldsymbol{F}(t) \cdot \boldsymbol{r} .
$$

Here $\boldsymbol{F}(t)$ is the electric field strength of the laser field, and the linear polarization is assumed. In this study, we use the laser pulses with the sine-squared envelope:

$$
\boldsymbol{F}(t)=\boldsymbol{F}_{0} \sin ^{2} \frac{\pi t}{T} \sin \omega_{0} t,
$$

where $T$ and $\omega_{0}$ denote the pulse duration and the carrier frequency, respectively; $F_{0}$ is the peak field strength. In all our calculations, we use the laser wavelength $800 \mathrm{~nm}\left(\omega_{0}=\right.$ $1.55 \mathrm{eV}$ ) and the pulse duration of 20 optical cycles (full width at half maximum is about $27 \mathrm{fs}$ ).

For TDDFT energy functional, we adopt the adiabatic approximation. That means the time-dependent single-particle potential $V_{\sigma}^{0}(\boldsymbol{r}, t)$ is defined by the same expression (17) as in the time-independent case but with the time-dependent electron densities. The initial values $(t=0)$ of Kohn-Sham spin orbitals and unperturbed (field-free) Hamiltonian come from solution of Eq. (14). Originally, a real (not complexscaled) potential $V_{\sigma}^{0}(\boldsymbol{r}, 0)$ is obtained on the GPS grid. In the time-dependent ECS scheme, this original numerical potential is used in the interior domain $r \leqslant R_{0}=R\left(x_{0}\right)$. In the exterior domain $r>R_{0}$, an analytical approximation is applied:

$$
V_{\sigma}^{0}(\boldsymbol{r}, 0)=\frac{1}{r}\left(-1+\frac{c_{1}}{r}+\frac{c_{2}}{r^{2}}\right),
$$

which can be readily complex scaled on the smooth ECS contour in the complex $r$ plane. Coefficients $c_{1}$ and $c_{2}$ are obtained by matching the numerical potential with the analytical approximation on the last two grid points in the interior domain. Note that Eq. (25) preserves the correct asymptotic behavior of the potential $(\sim-1 / r)$.

The total Hamiltonian of Eq. (22) can be represented as a sum of the unperturbed Hamiltonian $H_{0}$ and interaction $V(t)$ term due to the external field:

$$
\begin{gathered}
H(t)=H_{0}+V(t), \\
H_{0}=-\frac{1}{2} \nabla^{2}+V_{\sigma}^{0}(\boldsymbol{r}, 0), \\
V(t)=V_{\sigma}^{0}(\boldsymbol{r}, t)-V_{\sigma}^{0}(\boldsymbol{r}, 0)+v_{\mathrm{ext}}(\boldsymbol{r}, t) .
\end{gathered}
$$

To propagate the Kohn-Sham spin orbitals in time, the shortterm propagator

$$
U(t, \Delta t)=\exp \left\{-i \Delta t\left[H_{0}+V\left(t+\frac{1}{2} \Delta t\right)\right]\right\}
$$

is applied sequentially from $t=0$ to $t=T$. The smaller $\Delta t$, the more accurate is time propagation. The calculation of $U(t, \Delta t)$ at each time step is greatly facilitated by the second-order split-operator formula:

$$
\begin{aligned}
U(t, \Delta t)= & \exp \left[-i \frac{1}{2} \Delta t H_{0}\right] \exp \left[-i \Delta t V\left(t+\frac{1}{2} \Delta t\right)\right] \\
& \times \exp \left[-i \frac{1}{2} \Delta t H_{0}\right] .
\end{aligned}
$$

Here the field-free propagator $\exp \left[-i(1 / 2) \Delta t H_{0}\right]$ is timeindependent and calculated only once using the spectral expansion:

$$
\exp \left[-i \frac{1}{2} \Delta t H_{0}\right]=\sum_{k} \exp \left[-i \frac{1}{2} \Delta t E_{k}\right]\left|\psi_{k}^{R}\right\rangle\left\langle\psi_{k}^{L}\right|,
$$

where $E_{k}$ are the complex eigenvalues of the non-Hermitian ECS Hamiltonian $H_{0}$. The right eigenvectors $\psi_{k}^{R}$ and left eigenvectors $\psi_{k}^{L}$ are subject to biorthogonality and 
normalization condition:

$$
\left\langle\psi_{k^{\prime}}^{L} \mid \psi_{k}^{R}\right\rangle=\delta_{k^{\prime} k}
$$

The external field propagator $\exp \left[-i \Delta t V\left(t+\frac{1}{2} \Delta t\right)\right]$ is time dependent and must be calculated at each time step; however, upon GPS discretization it is represented by a diagonal matrix, and its computation is not time consuming. To avoid numerical instabilities related to the complex-scaled long-range dipole term, the interaction with the external field $V(t)$ in the propagator is restricted to the interior domain only, $r \leqslant R_{0}$.

\section{MULTIPHOTON IONIZATION AND HIGH-ORDER-HARMONIC GENERATION OF ARGON ATOMS}

\section{A. MPI probabilities and rates}

The time-dependent electron spin densities and total electron density in the interior domain are calculated according to the equations similar to those in the time-independent case:

$$
\begin{aligned}
& \rho_{\sigma}(\boldsymbol{r}, t)=\sum_{n=1}^{N_{\sigma}}\left|\psi_{n \sigma}(\boldsymbol{r}, t)\right|^{2}, \\
& \rho(\boldsymbol{r}, t)=\rho_{\uparrow}(\boldsymbol{r}, t)+\rho_{\downarrow}(\boldsymbol{r}, t) .
\end{aligned}
$$

Since the ECS Hamiltonian is non-Hermitian, the time propagation is not unitary. For any spin orbital, the normalization integral within the sphere $r \leqslant R_{0}$ decreases in time. One can calculate the time-dependent survival probability $P_{\mathrm{S}}(t)$ (probability that the atom is not ionized at time $t$ ) as follows:

$$
P_{\mathrm{s}}(t)=\prod_{n, \sigma} \int_{r \leqslant R_{0}} d^{3} r\left|\psi_{n \sigma}(\boldsymbol{r}, t)\right|^{2} .
$$

The time-dependent ionization probability $P_{\mathrm{i}}(t)$ and ionization rate $\Gamma(t)$ can be obtained according to

$$
\begin{gathered}
P_{\mathrm{i}}(t)=1-P_{\mathrm{s}}(t), \\
\Gamma(t)=-\frac{d}{d t} \ln P_{\mathrm{s}}(t) .
\end{gathered}
$$

We have calculated MPI probabilities and rates of $\mathrm{Ar}$ for several peak intensities of the laser field in the range $1 \times 10^{14} \mathrm{~W} / \mathrm{cm}^{2}$ to $5 \times 10^{14} \mathrm{~W} / \mathrm{cm}^{2}$. Besides the all-electron TDDFT analysis, we have also performed frozen-core model potential calculations which do not take into account dynamic multielectron response of the atom to the external field. In the latter case, the electronic motion is influenced by the static potential $V_{\sigma}^{0}(\boldsymbol{r})$, Eq. (17), and time-dependent external field $v_{\text {ext }}(\boldsymbol{r}, t)$, Eq. (23), only. This is similar to one-electron models which are frequently used in strong-field calculations of multielectron targets. In our present approach, however, we propagate in time all spin orbitals which represent a multielectron noninteracting system through a Slater determinant. Consequently, the Pauli principle is satisfied, and there are no spurious transitions to occupied one-electron states. We should also note that the initial unperturbed systems are the same in the frozen-core model and TDDFT, thus the energy spectrum and ionization potential are also the same. After the laser pulse, the system is still the same in the frozen-core model but may have changed in TDDFT if substantial excitation and/or ionization occur.

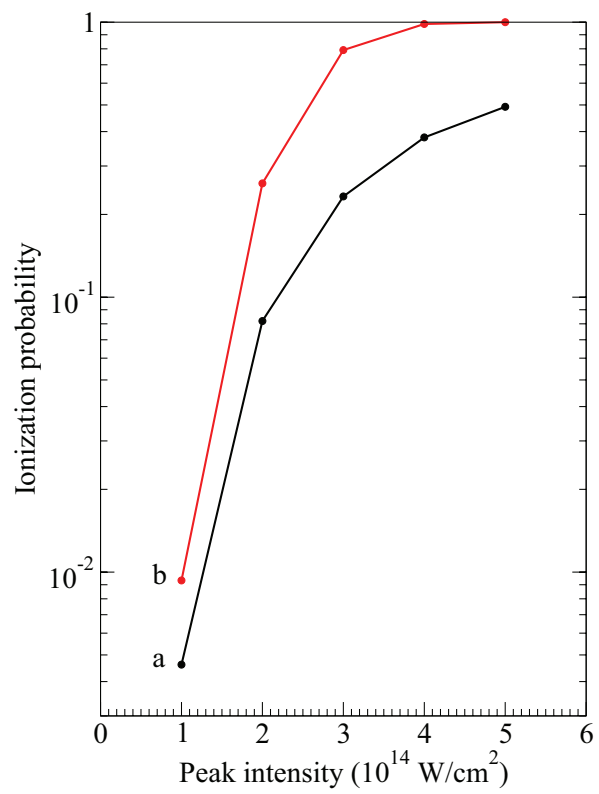

FIG. 2. (Color online) Ionization probability of Ar versus the peak intensity of the laser pulse: (a) TDDFT results; (b) frozen-core model potential calculations.

In Fig. 2, we show the intensity dependence of the MPI probabilities at the end of the laser pulse $(t=T)$. As one can see, the frozen-core results significantly overestimate those obtained by TDDFT. At the intensity $4 \times 10^{14} \mathrm{~W} / \mathrm{cm}^{2}$, according to the frozen-core model, Ar atoms get ionized with $98 \%$ probability; TDDFT calculations give a much smaller ionization probability of $38 \%$. To explain this difference qualitatively, we note that the TDDFT approach does contain dynamic response of the electrons to the external field and incorporates the polarization effects. Because of the polarization of the core, the effective time-dependent electric field acting upon the electron is weaker than that of the laser. Accordingly, the MPI probability obtained by TDDFT is smaller than the frozen-core model result which does not take the polarization of the core into account. An important role of multielectron polarization effects in strong-field ionization of Ar was recently demonstrated by the experiment on attosecond angular streaking and accompanying calculations within the tunneling ionization model in parabolic coordinates [28].

In Fig. 3, we present the time-dependent MPI rate for the peak intensity of the laser field $2 \times 10^{14} \mathrm{~W} / \mathrm{cm}^{2}$. In the upper panel, shown are the MPI rate and appropriately scaled squared electric field $F^{2}(t)$ [see Eq. (24)]. According to a simple picture of tunneling ionization, one can expect that the rate reaches its maximum at those times when the oscillating electric field of the laser is the strongest, twice per each optical cycle. As one can see from Fig. 3(a), this is the case for relatively weak fields only, at the beginning and at the end of the laser pulse. In the middle of the pulse, where the intensity is close to its peak value, the MPI rate and squared electric field oscillate out of phase. A similar picture is seen in our calculations for all the peak intensities higher or equal to $1 \times 10^{14} \mathrm{~W} / \mathrm{cm}^{2}$; only for the relatively weak laser pulse with the peak intensity $5 \times 10^{13} \mathrm{~W} / \mathrm{cm}^{2}$, the MPI rate oscillates in phase with the squared field at all times. To reveal the origin of 

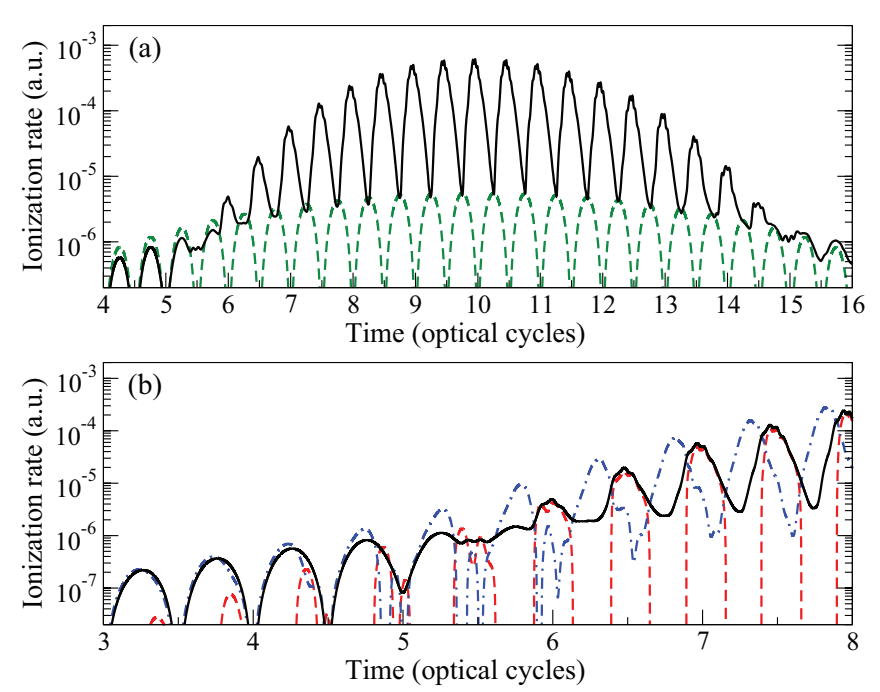

FIG. 3. (Color online) Time-dependent MPI rates of Ar for a $\sin ^{2}$ laser pulse with the carrier wavelength of $800 \mathrm{~nm}$ and duration of 20 optical cycles at the peak intensity $2 \times 10^{14} \mathrm{~W} / \mathrm{cm}^{2}$. (a) MPI rate (solid black line); scaled squared electric field (dashed green line). (b) Full MPI rate (solid black line); inner region ( $r \leqslant 10$ a.u.) contribution (dot-and-dashed blue line); outer region $(r>10$ a.u.) contribution (dashed red line).

this behavior, we split the survival probability $P_{\mathrm{s}}(t)$, Eq. (35), in two parts—one of them $\left[P_{\mathrm{s}}^{(1)}(t)\right]$ describing a close vicinity of the nucleus $(r \leqslant 10$ a.u. $)$, and another one $\left[P_{\mathrm{s}}^{(2)}(t)\right]$ - the remaining contributions to $P_{\mathrm{s}}(t)$ :

$$
\begin{gathered}
P_{\mathrm{s}}^{(1)}(t)=\prod_{n, \sigma} \int_{r \leqslant 10} d^{3} r\left|\psi_{n \sigma}(\boldsymbol{r}, t)\right|^{2}, \\
P_{\mathrm{s}}^{(2)}(t)=P_{\mathrm{s}}(t)-P_{\mathrm{s}}^{(1)}(t) .
\end{gathered}
$$

Then the MPI rate is also represented as a sum of two terms:

$$
\begin{gathered}
\Gamma(t)=\Gamma^{(1)}(t)+\Gamma^{(2)}(t), \\
\Gamma^{(1)}(t)=-\frac{1}{P_{\mathrm{s}}(t)} \frac{d P_{\mathrm{s}}^{(1)}(t)}{d t}, \\
\Gamma^{(2)}(t)=-\frac{1}{P_{\mathrm{s}}(t)} \frac{d P_{\mathrm{s}}^{(2)}(t)}{d t} .
\end{gathered}
$$

In Fig. 3(b), we plot $\Gamma(t)$ along with the contributions from the different spatial regions $\Gamma^{(1)}(t)$ and $\Gamma^{(2)}(t)$. The contribution from the inner region $\Gamma^{(1)}(t)$ always oscillates in phase with the squared electric field. For weaker fields (optical cycles 3-5), this contribution is dominant since the electron density in the outer region is small; the total MPI rate $\Gamma(t)$ follows $\Gamma^{(1)}(t)$ closely at these times. However, as the electric field becomes stronger (optical cycles 6-8) and the MPI rate increases, the electron density in the outer region increases too. Then the contribution of $\Gamma^{(2)}(t)$ becomes more significant, and the maxima of $\Gamma(t)$ coincide with those of $\Gamma^{(2)}(t)$. The latter are shifted in time with respect to the electric field because of the retardation effect for the electrons traveling from the vicinity of the nucleus to the outer region.

\section{B. HHG spectra}

To calculate the HHG spectra, we use a semiclassical approach, where the basic expressions come from the classical electrodynamics but the classical quantities such as dipole moment and its acceleration are replaced with the corresponding quantum expectation values. The spectral density of radiation energy can be expressed through the Fourier transforms of the acceleration $\boldsymbol{a}(t)$ or dipole moment $\boldsymbol{d}(t)$ [29]:

$$
\begin{gathered}
S(\omega)=\frac{2}{3 \pi c^{3}}|\widetilde{\boldsymbol{a}}(\omega)|^{2}=\frac{2 \omega^{4}}{3 \pi c^{3}}|\widetilde{\boldsymbol{d}}(\omega)|^{2} ; \\
\widetilde{\boldsymbol{a}}(\omega)=\int_{-\infty}^{\infty} d t \boldsymbol{a}(t) \exp (i \omega t), \\
\tilde{\boldsymbol{d}}(\omega)=\int_{-\infty}^{\infty} d t \boldsymbol{d}(t) \exp (i \omega t)
\end{gathered}
$$

( $c$ is the speed of light) and the expectation values of the dipole moment and acceleration are defined as follows:

$$
\begin{gathered}
\boldsymbol{d}(t)=\int d^{3} r \boldsymbol{r} \rho(\boldsymbol{r}, t) ; \\
\boldsymbol{a}(t)=-\int d^{3} r \nabla\left[v_{\mathrm{n}}(r)+v_{\mathrm{ext}}(\boldsymbol{r}, t)\right] \rho(\boldsymbol{r}, t) .
\end{gathered}
$$

They satisfy the same relation as the corresponding classical quantities:

$$
\frac{d^{2}}{d t^{2}} \boldsymbol{d}(t)=\boldsymbol{a}(t)
$$

The expression for $\boldsymbol{a}(t)$ can be derived from that for $\boldsymbol{d}(t)$ with the help of the Ehrenfest theorem. We note that only the nuclear and external field potentials are present in Eq. (47). When multielectron targets are treated exactly, the electron-electron interaction does not contribute to the expectation value of acceleration due to Newton's third law since the electrons are identical and have the same masses and charges. In TDDFT, that means the exact exchange-correlation potential (as well as the Hartree potential) does not contribute to the expectation value of acceleration [30]. For approximate exchange-correlation potentials, this is not always the case. Consequently, the dipole and acceleration forms of the HHG spectra (43) with the expectation values defined in Eqs. (46) and (47) are not necessarily identical in TDDFT. For the expectation value of acceleration, spatial integration in Eq. (47) emphasizes short distances; for the expectation value of dipole moment, spatial integration in Eq. (46) emphasizes long distances. In our calculations of the HHG spectra, we adopt the acceleration form. We assume it is more accurate since our numerical wave functions are more accurate at short distances due to denser spatial grid.

In Fig. 4, we present the HHG spectra for the laser peak intensity $2 \times 10^{14} \mathrm{~W} / \mathrm{cm}^{2}$; the upper panel contains the TDDFT spectrum, while the lower panel shows the results of the frozen-core model potential calculations. For the frozencore model, Eq. (47) for the expectation value of acceleration must contain the full model potential $V_{\sigma}^{0}(\boldsymbol{r})$, and not just the interaction with the nucleus. Although all the spin orbitals are being propagated in time and contribute to the time-dependent electron density in the frozen-core model, the HHG spectrum is strongly dominated by the highest-occupied $3 p(m=0)$ subshell. In this sense, the frozen-core model appears a singleactive-electron model. On the contrary, the TDDFT spectrum 


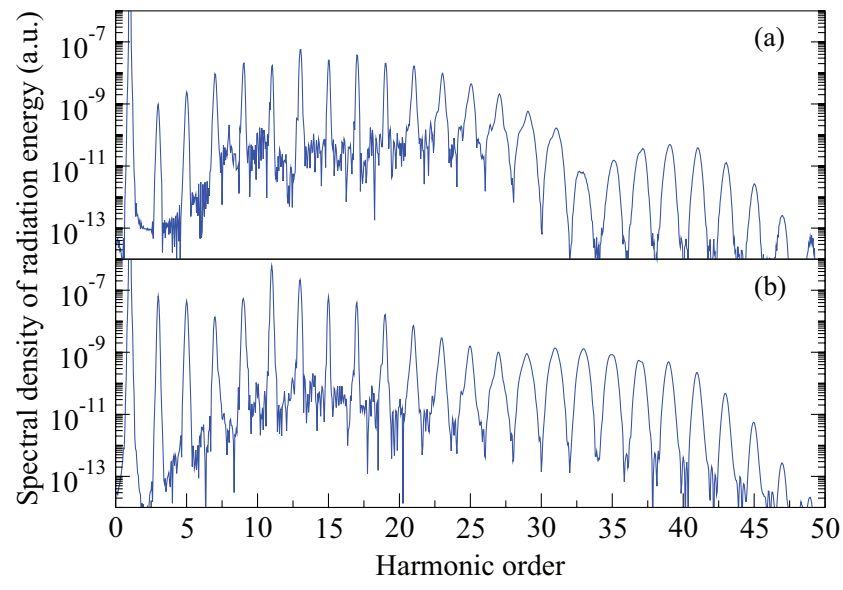

FIG. 4. (Color online) Spectral density of harmonic radiation energy for a $\sin ^{2}$ laser pulse with the carrier wavelength of $800 \mathrm{~nm}$ and duration of 20 optical cycles at the peak intensity $2 \times 10^{14} \mathrm{~W} / \mathrm{cm}^{2}$ : (a) TDDFT results; (b) frozen-core model potential calculations.

contains comparable contributions from different Kohn-Sham spin orbitals thus indicating the significance of multielectron effects. Both frozen-core model and TDDFT spectra have a minimum in the central part which is closely related to the Cooper minimum [31] observed in the photoionization cross sections of Ar. This minimum originates from the nodal structure of the $3 p$ wave function of Ar which causes the bound-continuum $3 p-E d$ dipole matrix element to vanish at some energy $E$ in the continuum [31]. According to the three-step model of HHG [32], the third step of this process is recombination of the recolliding electron with the core. Ionization and recombination are mutually inverse processes, hence a minimum in the photoionization cross section must have its reflection in the HHG spectra as well. The Cooper minimum in the HHG spectra of Ar has already been observed experimentally many times [33-37]. In our TDDFT calculations, this minimum is clearly seen in the vicinity of the 33rd harmonic $(\sim 51 \mathrm{eV})$. In the frozen-core model spectrum, the minimum is less pronounced and shifted to lower energies (about the 29th harmonic, $\sim 45 \mathrm{eV}$ ). We note that another one-electron model [38] also gives this minimum at lower energies $(\sim 40 \mathrm{eV})$. As one can see, dynamic multielectron response is quite important in shaping the Cooper minimum in the Ar HHG spectra.

Figure 5 shows the HHG spectra of Ar calculated by the TDDFT-ECS method at three different intensities: $2 \times$ $10^{14} \mathrm{~W} / \mathrm{cm}^{2}, 3 \times 10^{14} \mathrm{~W} / \mathrm{cm}^{2}$, and $4 \times 10^{14} \mathrm{~W} / \mathrm{cm}^{2}$. As one can see, with increasing intensity, the cutoff of the HHG spectrum shifts to higher energies in fair agreement with the well-known semiclassical law [39] $\left(E_{\mathrm{i}}+3.17 U_{\mathrm{p}}, E_{\mathrm{i}}\right.$ and $U_{\mathrm{p}}$ being the ionization energy and the ponderomotive potential, respectively). At the same time, the position of the Cooper minimum in the spectrum appears intensity independent and corresponds approximately to $51 \mathrm{eV}$ (33rd harmonic), in good accord with the experimental observations [34-36] (51-54 eV). As was noticed in Refs. [35,36], the measured minimum in the HHG spectra is somewhat shifted to higher energies compared with the measurements in the photoionization [40] and electronic excitation [41] experiments ( $48 \mathrm{eV}$ and

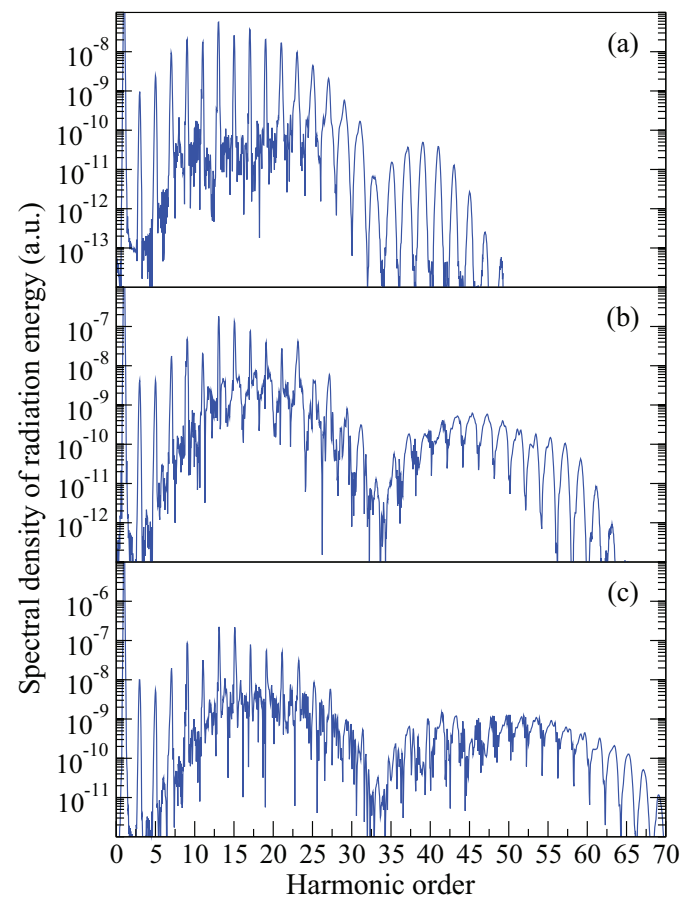

FIG. 5. (Color online) Spectral density of harmonic radiation energy for a $\sin ^{2}$ laser pulse with the carrier wavelength of $800 \mathrm{~nm}$ and duration of 20 optical cycles: (a) peak intensity $2 \times 10^{14} \mathrm{~W} / \mathrm{cm}^{2}$; (b) peak intensity $3 \times 10^{14} \mathrm{~W} / \mathrm{cm}^{2}$; (c) peak intensity $4 \times 10^{14} \mathrm{~W} / \mathrm{cm}^{2}$.

$50 \mathrm{eV}$, respectively). Although ionization and recombination are mutually inverse processes, an experimentally measured photoionization cross section contains an incoherent sum of two contributions ( $p \rightarrow s$ and $p \rightarrow d$ channels), while in the HHG recombination amplitude a coherent sum of these contributions is present [36]. This circumstance can partly explain the blueshift of the Cooper minimum in the HHG spectrum $[35,36]$. We also point out the multielectron effects and dynamic core polarization; their importance is clearly seen from the comparison of our TDDFT and frozen-core model calculations.

\section{SUMMARY}

In this paper, we have presented the TDDFT-ECS method for treatment of multielectron atoms subject to laser fields. We have shown that exterior complex scaling can be successfully implemented in the framework of time-dependent densityfunctional theory. It ensures that the outgoing-wave boundary conditions are imposed on the wave function at large distances; this is important for correct description of ionization caused by external time-dependent fields. When combined with the generalized pseudospectral discretization, TDDFT-ECS method provides an accurate and efficient computational scheme for calculations of multiphoton processes in multielectron atoms. We have applied the method for calculations of MPI and HHG in Ar. Our results show that dynamic multielectron response is extremely important in both processes. The calculations performed using a static single-electron model potential, without taking multielectron effects into account, significantly overestimate the MPI probabilities compared with those obtained by TDDFT for the same parameters of the 
laser field. In the HHG spectra of Ar, the effect of multiple electronic shells is clearly seen in shaping of the Cooper minimum. In the TDDFT-ECS calculations, this minimum appears approximately at the photon energy of $51 \mathrm{eV}$, in good agreement with the experimental observations, while in the static potential model the minimum is less pronounced and shifted to lower energies.

\section{ACKNOWLEDGMENT}

This work was partially supported by the Chemical Sciences, Geosciences and Biosciences Division of the Office of Basic Energy Sciences, Office of Sciences, US Department of Energy. We also acknowledge the partial support of St. Petersburg State University (Grant No. 11.38.654.2013).
[1] F. Krausz and M. Ivanov, Rev. Mod. Phys. 81, 163 (2009).

[2] J. Itatani, J. Levesque, D. Zeidler, H. Niikura, H. Pépin, J. C. Kieffer, P. B. Corkum, and D. M. Villeneuve, Nature (London) 432, 867 (2004).

[3] O. Smirnova, Y. Mairesse, S. Patchkovskii, N. Dudovich, D. Villeneuve, P. Corkum, and M. Y. Ivanov, Nature (London) 460, 972 (2009).

[4] S. Haessler, J. Caillat, W. Boutu, C. Giovanetti-Teixeira, T. Ruchon, T. Auguste, Z. Diveki, P. Breger, A. Maquet, B. Carré, R. Taïeb, and P. Saliéres, Nat. Phys. 6, 200 (2010).

[5] A. D. Shiner, B. E. Schmidt, C. Trallero-Herrero, H. J. Wörner, S. Patchkovskii, P. B. Corkum, J. C. Kieffer, F. Légaré, and D. M. Villeneuve, Nat. Phys. 7, 464 (2011).

[6] M. J. Nandor, M. A. Walker, L. D. Van Woerkom, and H. G. Muller, Phys. Rev. A 60, R1771 (1999).

[7] H. G. Muller, Phys. Rev. A 60, 1341 (1999).

[8] S. I. Chu, J. Chem. Phys. 123, 062207 (2005).

[9] D. A. Telnov and Shih-I Chu, Phys. Rev. A 79, 041401(R) (2009).

[10] D. A. Telnov and Shih-I Chu, Phys. Rev. A 80, 043412 (2009).

[11] J. Heslar, D. A. Telnov, and Shih-I Chu, Phys. Rev. A 83, 043414 (2011).

[12] E. Balslev and J. M. Combes, Commun. Math. Phys. 22, 280 (1971).

[13] A. Aguilar and J. M. Combes, Commun. Math. Phys. 22, 265 (1971).

[14] C. A. Nicolaides and D. R. Beck, Phys. Lett. A 65, 11 (1978).

[15] B. Simon, Phys. Lett. A 71, 211 (1979).

[16] A. Palacios, C. W. McCurdy, and T. N. Rescigno, Phys. Rev. A 76, 043420 (2007).

[17] D. A. Horner, C. W. McCurdy, and T. N. Rescigno, Phys. Rev. A 71, 012701 (2005).

[18] C. W. McCurdy, M. Baertschy, and T. N. Rescigno, J. Phys. B 37, R137 (2004).

[19] C. W. McCurdy, C. K. Stroud, and M. K. Wisinski, Phys. Rev. A 43, 5980 (1991).
[20] C. W. McCurdy, D. A. Horner, and T. N. Rescigno, Phys. Rev. A 65, 042714 (2002).

[21] D. A. Telnov and Shih-I Chu, Phys. Rev. A 59, 2864 (1999).

[22] D. A. Telnov and Shih-I Chu, Phys. Rev. A 66, 043417 (2002).

[23] S. I. Chu and D. A. Telnov, Phys. Rep. 390, 1 (2004).

[24] D. A. Telnov and Shih-I Chu, Phys. Rev. A 71, 013408 (2005).

[25] D. A. Telnov and Shih-I Chu, Phys. Rev. A 76, 043412 (2007).

[26] R. van Leeuwen and E. J. Baerends, Phys. Rev. A 49, 2421 (1994).

[27] K. Sevier, At. Data Nucl. Data Tables 24, 323 (1979).

[28] A. N. Pfeiffer, C. Cirelli, M. Smolarski, D. Dimitrovski, M. Abu-samha, L. B. Madsen, and U. Keller, Nat. Phys. 8, 76 (2012).

[29] L. D. Landau and E. M. Lifshitz, The Classical Theory of Fields (Pergamon Press, Oxford, 1975).

[30] G. Vignale, Phys. Rev. Lett. 74, 3233 (1995).

[31] J. W. Cooper, Phys. Rev. 128, 681 (1962).

[32] M. Lewenstein, P. Balcou, M. Y. Ivanov, A. L'Huillier, and P. B. Corkum, Phys. Rev. A 49, 2117 (1994).

[33] S. Minemoto, T. Umegaki, Y. Oguchi, T. Morishita, A. T. Le, S. Watanabe, and H. Sakai, Phys. Rev. A 78, 061402(R) (2008).

[34] H. J. Wörner, H. Niikura, J. B. Bertrand, P. B. Corkum, and D. M. Villeneuve, Phys. Rev. Lett. 102, 103901 (2009).

[35] J. P. Farrell, L. S. Spector, B. K. McFarland, P. H. Bucksbaum, M. Gühr, M. B. Gaarde, and K. J. Schafer, Phys. Rev. A 83, 023420 (2011).

[36] J. Higuet, H. Ruf, N. Thiré, R. Cireasa, E. Constant, E. Cormier, D. Descamps, E. Mével, S. Petit, B. Pons, Y. Mairesse, and B. Fabre, Phys. Rev. A 83, 053401 (2011).

[37] X. Wang, M. Chini, Q. Zhang, K. Zhao, Y. Wu, D. A. Telnov, Shih-I Chu, and Z. Chang, Phys. Rev. A 86, 021802(R) (2012).

[38] A. T. Le, T. Morishita, and C. D. Lin, Phys. Rev. A 78, 023814 (2008).

[39] P. B. Corkum, Phys. Rev. Lett. 71, 1994 (1993).

[40] J. A. R. Samson and W. C. Stolte, J. Electron Spectrosc. Relat. Phenom. 123, 262 (2002).

[41] W. F. Chan, G. Cooper, X. Guo, G. R. Burton, and C. E. Brion, Phys. Rev. A 46, 149 (1992). 